\title{
Millimeter and submillimeter wave technology developments for the next generation of fusion devices
}

\author{
R. J. Hwu, L. Sjogren, and N. C. Luhmann, Jr. \\ University of California, Depariment of Electrical Engineering, Los Angeles, California 90029 \\ Z. Popovic, R. W. Weikel, M. Kim, and D. B. Rutledge \\ California Institute of Technology, Division of Engineering and Applied Science, Pasadena, \\ California 91125
}

(Presented on 10 May 1990)

There is increasing demand for compact watt-level coherent sources in the millimeter and submillimeter wave region. The approach that we have taken to satisfy this need is to fabricate two-dimensional grids loaded with oscillators, electronic beam steerers, and frequency multipliers for quasioptical coherent spatial combining of the outputs of a large number of low-power devices.

\section{INTRODUCTION}

Millimeter and submillimeter wave diagnostics have proven to be invaluable for the determination of fusion plasma parameters. The successful development of new fasion devices such as CIT and ITER requires the use of watt-level sources as probe beams and as local oscillators for the arrays. Although electron beam devices such as backward wave oscillators (BWOs) can readily supply the power requirements, their size, expense, and lifetime have limited their applications. The approach that we have taken is to employ solid-state microfabrication techniques. To satisfy the size and lifetime constraints, we have thus far concentrated on solid state power sources. To provide the required power levels, we have employed quasioptical spatial power combining of the outputs of large planar arrays of devices. Here, it should be noted that this approach to power combining significantly reduces the losses as compared to conventional waveguide approaches. The development effort involves oscillator grids, frequency multiplier arrays, beam steering arrays, and mixer arrays. The eventual goal is the development of a millimeter wave "television camera" as illustrated in Fig. 1.

\section{SCHOTTKY DIODE×GRID DOUBLER ARRAY}

The concept of a monolithic diode-grid doubler array was implemented in a proof-of-principle experiment with Schottky diode grids fabricated on GaAs wafers. "The grid period is chosen to be about half the dielectric wavelength to avoid exciting substrate modes. Vertical metal strips serve as inductive leads for the diodes, while horizontal strips permit biasing the diodes. A second harmonic conversion efficiency of $9.5 \%$ and output powers of $0.5 \mathrm{~W}$ were achieved at $66 \mathrm{GHz}$. The average series resistance was $27 \Omega$ and the minimum capacitance was $18 \mathrm{fF}$ at a reverse breakdown voltage of $-3 \mathrm{~V}$. The measurements indicate that the diode grid is a feasible device for generating watt-ievel powers at millimeter and submillimeter frequencies, and that substantial improvement is possible by increasing the diode breakdown voltage and reducing the series resistance. The theoretical model provides confidence in predictions of achievable cw output power levels of $2.5 \mathrm{~W}$ at a frequency of $188 \mathrm{GHz}$ with an edge-cooled grid containing 1000 diodes. Such an array is currently being fabricated.

\section{H. SCHOTTKY DIODE-GRID SHASE-SHFTER ARRAY}

The same Schottky diode-grid was also used for phase shift measurements ${ }^{2}$ with the eventual goal being an electronic beam steering array. It has been shown that a complete 360 degree range of phase control could be obtained by use of a two-layer stack of properly designed diode grids as shown in Fig. 2, where there is a refiecting layer on the back and a quartz matching layer on the front (see Fig. 3 ). With such a structure, beam steering can be accomplished in the direction perpendicular to the diode rows by use of row biases that produce a linear progressive phase shift. Initial proof-of-principle tests were performed on single diode grids described in the preceding section. Here, a phase shift of $70^{\circ}$ with a $7 \mathrm{~dB}$ loss was obtained at $93 \mathrm{GHz}$ when the bias on the diode grid was changed from -3 to $1 \mathrm{~V}$ in agreement with theory. Using the parameters of the diodes currently under fabrication, we anticipate signifcant improved performance for a full two layer structure. Using a reailistic dioce resistance of $10 \Omega$, a refiection loss of $2.5 \mathrm{~dB}$ is predicted. Bandwidth, which will be limited by scan angle, should be about $7 \%$ for a $45^{\circ}$ scan angle.

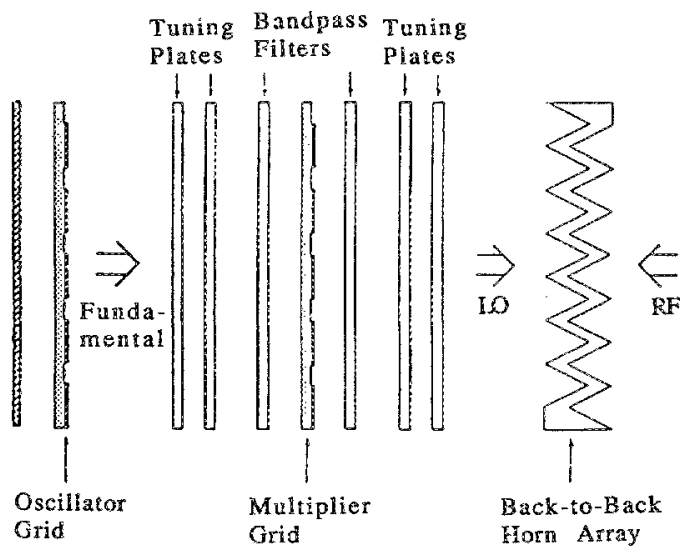

FIG. 1. Schematic of millimeter wave "television camera." 

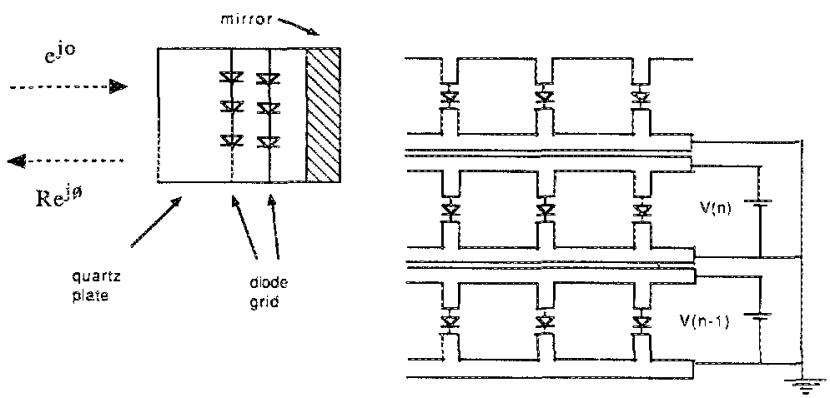

FIG. 2. Schematic of two-layer phase shifter and diode-grid layout.

\section{BARRIER-INTRINSIC $-N^{+}$DIODE-GRID TRIPLER ARRAY}

In an extension of the GaAs Schottky diode doubler array studies, a back-to-back configuration of BIN (barrier-intrinsic $-N^{+}$) diodes has been developed. ${ }^{3,4}$ The barrier-intrinsic $-N^{+}$(BIN) diode incorporates a thin $(1000 \AA)$ undoped semiconductor layer $(I)$ on a heavily doped layer $\left(N^{+}\right)$serving as a back contact. On top of the undoped layer there is an ultrathin $(100 \AA)$ electron-blocking barrier layer $(B)$ in contact with a metal top layer. This blocking layer can be formed by an insulator, a semiconductor with a very wide band gap, or a Mott barrier. The device can be switched rapidly between two capacitance states that correspond to accumulation of electrons at the barrier and depletion of the intrinsic layer, respectively, by the applied bias. This results in a highly nonlinear capacitance-voltage characteristic that is needed for efficient harmonic generation.

A novel concept that allows the construction of a GaAs BIN diode entirely by selective doping during $\mathrm{MBE}$ growth has recentiy been described. ${ }^{3}$ A Mott barrier is

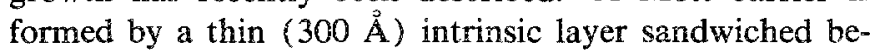
tween the top metal contact and a charge sheet created by selective doping.

Due to the blocking barrier of the BIN structure, two diodes can be operated back-to-back generating a sharp
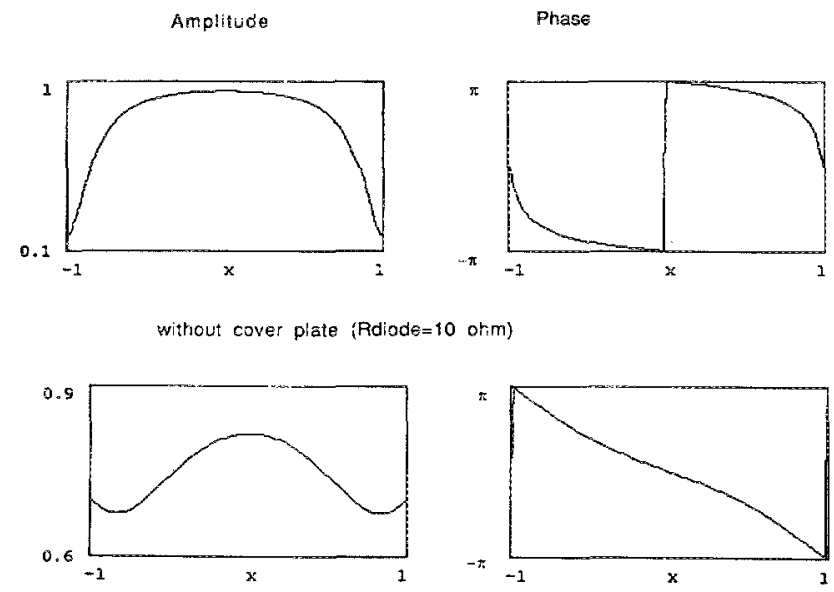

with cover plate (Rolode-10 ohm)

FIG. 3. Phase shift calculated for two-grid design.

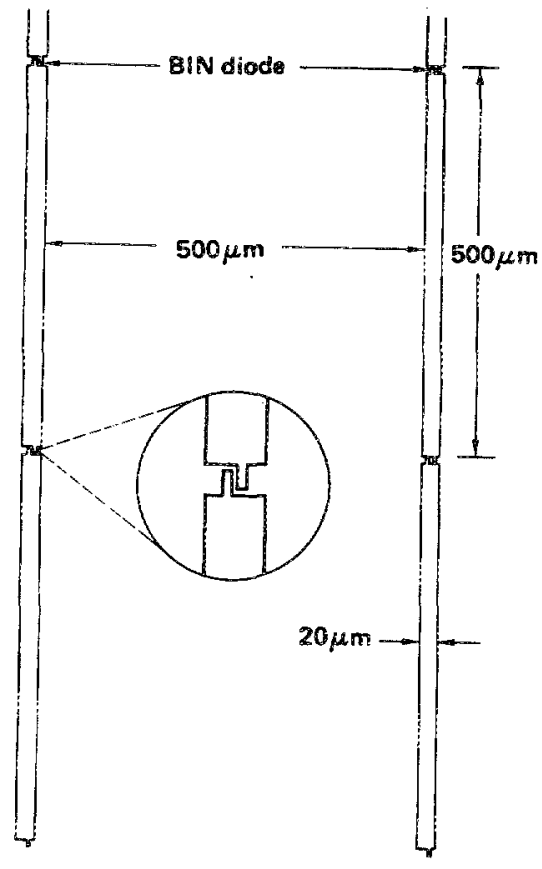

FIG. 4. BIN diode tripler array.

symmetric spike in the capacitance-voltage curve, which eliminates even harmonics and thus favors tripler and quintupler operation. ${ }^{4}$ The height and width of this capacitance-voltage curve can, in principle, be adjusted by doping control alone. This arrangement needs no external ohmic contact resulting in a highly efficient frequency tripler.

A novel grid structure has recently been designed for the BIN diode tripler array as shown in Fig. $4 .{ }^{4}$ The metal grid consists of a columnar mesh of aluminum strips with Schottky electrodes on each end. The small dimensions of the Schottky electrode area minimizes the zero-voltage capacitance and series resistance to increase the diode cutoff frequency. The two neighboring Schottky electrodes are designed to provide the back-to-back configuration. There is no need for dc bias lines in the diode-grid design. The grid requires only one metal pattern, which greatly facilitates the fabrication.

\section{GRID OSCILLATOR ARRAYS}

Quasioptical spatial power combining has been demonstrated with Gunn diodes ${ }^{5}$ and MESFETs. ${ }^{6,7}$ The oscillator configuration is shown in Fig. 5. The active grid, which is fabricated on a dielectric substrate, is placed in the transverse plane of a Fabry-Perot resonator. One of the reflectors of the resonators is partially transparent and serves as the cavity output coupler. When the devices are biased in their negative resistance region, they start oscillating at different frequencies, but the cavity provides feedback and the devices couple through the cavity and oscillate in a single resonator mode. It is important to reaiize that the locking does not require an external signal. This configuration is analogous to a laser, where the active medium is the two-dimensional grid, rather than the more 


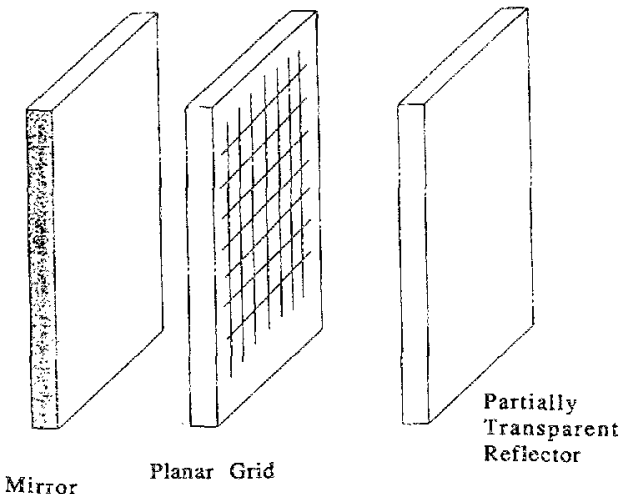

FIG. 5. Oscillator-grid resonator configuration.

familiar volume gain medium. Proof-of-principle measurements were performed on a 9-Gunn diode grid. ${ }^{5}$ The devices exhibited self-locking and power combining between 10 and $13 \mathrm{GHz}$. The total power was found to be proportional to the square of the number of devices.

An $X$-band hybrid model of a $25 \mathrm{MESFET}$ grid was fabricated on a quarter-wavelength- thick microwave substrate. Simple scaling should apply for higher frequencies. The oscillator configuration is the same as in Fig. 5. The vertical lines connected to the transistor gate and drain terminals support the if current flow, and the radiated electric field vector is parallel to them (the measured crosspolarized field was more than $20 \mathrm{~dB}$ below this polarization). The horizontal lines are used for dc bias. The maximum effective radiated power reiative to an isotropic source (ERP) was $37 \mathrm{~W}$ at $9.7 \mathrm{GHz}$. The actual radiated power from the grid was $464 \mathrm{~mW}$, which is 25 times the power typically generated with these MESFETs in single transistor microstrip circuits. The de to rf conversion efficiency was $14.5 \%$.

The second MESFET grid oscillator eliminated the dielectric substrate, ${ }^{7}$ as illustrated in Fig. 6. This grid consists of $36(6 \times 6)$ MESFET elements that are solded directly onto a stack of metal bars. The bars provide excellent heatsinking and de bias, and they act as a partially transparent reflector as well. This grid oscillates around $3 \mathrm{GHz}$. The measured ERP is $3.0 \mathrm{~W}$. This gives a dc to rf efficiency of 22 , and an isotropic conversion gain of $4.8 \mathrm{~dB}$.

The third hybrid grid oscillator was a planar 100element oscillator at $5 \mathrm{GHz}$, as shown in Fig. 5. This grid is made on a dielectric substrate, and the mirror position can be changed. The grid itself is the partially transparent refiector. This grid demonstrates the possibility of waferscale monolithically integrated high-power grid oscillators.

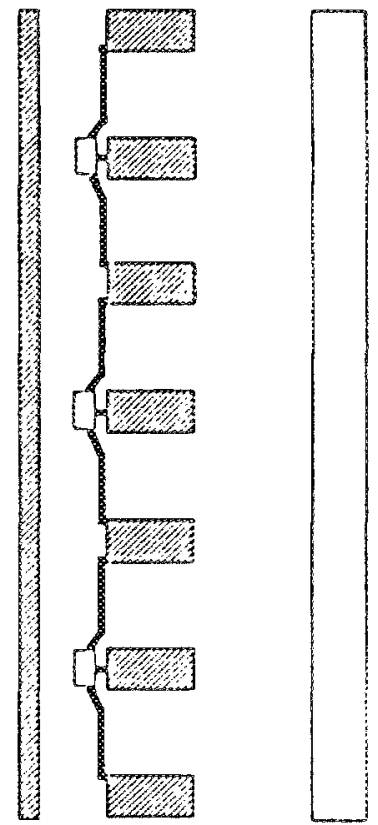

FIG. 6.6 by 6 MESFET grid.

\section{SUMMARY AND CONCLUSIONS}

Theoretical and experimental studies of monolithic planar frequency multiplier, electronic beam steering, and oscillator arrays have demonstrated the viability of the quasioptical spatial power combining approach. Current directions include the study of advanced blocking barrier multiplier structures, monolithic oscillator arrays and the development of two-dimensional $360^{\circ}$ electronic beam steering arrays. Oscillator grids operating up to $100 \mathrm{GHz}$ employing both two- and three-terminal devices are anticipated with the muliplier arrays expected to produce aseful power at frequencies up to $1 \mathrm{THz}$, thereby satisfy the needs of high-field, high-density devices such as CIT.

\section{ACKNOWLEDGMENTS}

This work was supported by the US Army Research Office, the US Department of Energy, Northrop, TRW, and the California MLRO Program.

'C. F. Jou, W. W. Lam, H. Chen, K. Stolt, N. C. Luhmann, Jr., and D. B. Rutledge, IEEE Trans. Microwave Theory Tech. 36, 1507 (1988).

${ }^{2}$ W. W. I.am, C. F. Jou, H. Chen, K. Stolt, N. C. Luhmann, Jr., and D. B. Rutledge, IEEE Trans. Microwave Theory Tech. 36, 902 (1987).

${ }^{3}$ U. Lieneweg, B. R. Hancock, and J. Maseriian, 12th International Conference on Infrared and Millimeter Waves, Tech. Dig., pp. 6-7 (1987).

${ }^{4}$ R. J. Hwu, C. F. Jou, N. C. Luhmann, Jr., W. W. Lam, D. B. Ruthedge, B. Hancock, U. Lieneweg, and J. Maserjian, Rev. Sci. Instrum. 59, 1577 (1988).

${ }^{5}$ Z. B. Popovic and D. B. Rutledge, 1988 IEEE Antemas and propagation Symposium, June 1988.

${ }^{\circ}$ Z. B. Fopovic, M. Kim, and D. B. Rutledge, Int. J. Infrared Millimeter Waves 9, 647 (1988).

${ }^{7}$ Z. B. Popovic, R. M. Weikle, M. Kim, K. A. Potter, and D. B. Rutledge, IEEE Trans. Microwave Theory Tech. 38, 225 (1990). 